\title{
The Research on Forensic DNA Evidence Base on Criminal Law Vision
}

\author{
Changmeng Jiang \\ Xi'an Politics Institute of PLA, Xi'an, China \\ 294244476@qq.com
}

Keywords: DNA evidence; Procedural Safeguards; Criminal proceedings

\begin{abstract}
Since the introduction of DNA technology into the field of forensic science, it has gradually become the main means of forensic evidence identification in criminal cases. The forensic DNA evidence produced by this technology has important functions in criminal proceedings, which can provide clues for the investigation and provide the basis for the identification of criminals. However, because of the limitations of the scientific technology, DNA evidence in the criminal justice function has not been fully played, there are still many problems in the application. Therefore, in the criminal procedure to standardize the collection and detection of DNA evidence, improve the DNA evidence in court review procedures, in order to better play its role in the court trial.
\end{abstract}

\section{Introduction}

In 1985, professor jeffreys published a paper in nature, which was considered the first time to introduce a forensic DNA analysis technique. With the appearance of a series of DNA extraction technology, we are very convenient for DNA acquisition, DNA identification and analysis technology has promoted the application of DNA in many fields, the significance of which is the application of forensic material evidence detection. the application and development of this technology has created a new way for forensic material evidence detection, which greatly accelerated the development of forensic medicine and criminal cases. Since then, forensic DNA testing technology has begun to develop rapidly.

The most widely used forensic DNA testing technology in the field of criminal justice is DNA fingerprinting. The principle of this technology is that human nucleotide sequence is different, with the restriction enzyme digestion, can produce different length of DNA fragments, this kind of different length fragments have polymorphism in the population. There are two kinds of DNA fingerprinting technology, which are divided into multi - site and unit - point probes. multi - site probes can simultaneously detect multiple loci. the characteristics of the four characteristics of high identification probability can basically achieve the purpose of individual identification, but it has complex operation, long detection cycle, higher requirements for forensic samples. But the unit point probe only has one detection site, the identification efficiency is poor, with the development and progress of DNA analysis technology, it has been gradually replaced by str - PCR technology. Str - PCR has a variety of advantages, first of all, the sensitivity of this detection method is high, the amplification yield of the two bands is equal, and secondly because of the wide distribution of str loci in the genome, so we can detect some of the degradation DNA, for some incomplete forensic evidence, this method can be a good help detection and analysis. However, str technology also has some disadvantages, because of its high sensitivity, so once the forensic evidence is mixed with other DNA, the instant is trace, will also cause the sample pollution, leading to wrong judgment. But in general, the str - PCR technology greatly improves the identification degree of human. in recent years, with the rapid development of PCR amplification technology and the emergence of automatic sequencing equipment, the sensitivity and recognition rate of str are higher.

In the criminal procedure, the forensic expert examines the biological samples from the detection organs, and uses the forensic DNA testing technology to analyze the consistency and the genetic after the conclusion of the judgment is the forensic DNA evidence. Because there is a large number of biological evidence containing DNA molecules in the crime scene, the information about the cases and the criminals can be obtained through the forensic DNA testing of the trace biological 
samples ignored or not easily eliminated by these criminals. In addition, because DNA molecules live longer, even in those suspected unsolved problems, DNA can be detected in bone or body fluids in a number of years of crime. This makes the method of DNA detection more widely used than traditional methods, and is far more reliable than previous methods of laboratory analysis of biological evidence.

\section{The Function of Forensic DNA Evidence}

To Analyze the Case, Determine whether He or She Commits Suicide, so as to Provide a basis for the Investigation of the Case, and to Ensure that the Investigation Work is Carried out in the Right Direction. Criminal cases can not be reproduced as what has happened. This not determines its complexity. Materialist dialectics believes that the human cognitive ability is limited, investigators may make the wrong judgment for a series of reasons in the preliminary identification of the case. Sometimes, the incident of normal death may be investigated as a criminal case to waste human and material resources. In some cases, real criminal cases may also be dealt with as suicide cases, resulting in impunity for criminals. In some cases, the use of forensic DNA testing techniques can help investigators analyze the nature of the case and specify the direction for the next step.

To Deduce if the offender is Injured and to Narrow the Scope of the Investigation. Blood is a special mark of the high incidence of homicide cases. By testing the blood blood on the spot, it can be concluded that whether the victim is injured or not in the course of the crime can provide clues for the investigation department, so as to reduce the scope of the investigation, save time for the investigation organs and improve work efficiency. For example, in a month in 2015, a city in Jingzhou city, a bungalow in a bungalow, a family of elderly women, a number of cash stolen in the house. The scene investigators extracted a fingerprint from the spot on the spot and found the DNA suspected to be different from the victim. Yigeyuehou, ja,. After DNA tests, the victim's lost flashlight on the victim's flashlight, the damaged necklace of the victim's DNA, and the victim's lost necklace, DNA typing and the victim's DNA were examined. At this point, DNA technology determines that such a person has a major suspect. The investigators accordingly arrested the suspect, and so - and - so.

By Finding Corpse to Provide Clues to the Investigation. Determining the identity of the dead is a common problem in criminal investigation. only the identification of the identity of the deceased can carry out the next step of investigation around the social relationship of the deceased. However, in the actual work, the unknown corpses encountered by investigators are mostly corpses floating corpses, body bodies, charred bodies, carrion, corpses or highly decayed, or the body is completely destroyed by carbon black, or unable to find the whole body, or because of the long time, only a pile of bones remain, in which case the families of the deceased are also difficult to identify. With the help of forensic DNA testing techniques, such as the identification of suspicious parents or children of the anonymous skeleton, it can help investigators identify the identity of the deceased.

Determine the Nature of the Case and Specify the Direction for the Investigation. The forensic DNA testing technology can effectively identify the mixed samples, which is mainly used in the case analysis and the determination of the nature of the cases in the criminal investigation, through the inspection of the mixed spot samples collected on the scene, to determine whether the victim was raped or raped. A homicide in a broken house on a mountain in a tourist area. The entity has been highly decayed, and the vaginal extract extracted from the body was tested positive by acid phosphatase test. The genotypes of sperm DNA were not detected by forensic DNA test. On the spot, a lot of toilet paper containing mixed sperm stains was extracted. through the forensic DNA test, different kinds of sperm from the mixed sperm were detected. through the examination of the female components in mixed sperm, and compared with the deceased, it was found that the female components of mixed sperm in two kinds of toilet paper were the same as the DNA genotype of the deceased, so the case was considered as rape homicide. The latter investigation organs according to the detection of the criminal suspects of the DNA genotyping of the case.

Paternity is Determined by Forensic DNA Testing to Provide Clues to the Suspect. By examining the DNA of progeny and parents, it is possible to determine whether the offspring and 
parents have parental power. Since the DNA of each human cell is derived from both parents, paternity testing can be performed through the triple test of the mother - son. In some cases involving parent-child relationship, through the use of this technology, can identify the personnel who may be related to the case, investigators use this clue can lock the suspect, and finally solve the case.

Determining the Time of Death of the Deceased and Providing Assistance for the Timely Detection of the Case. In the investigation practice, to determine the dead time of the dead plays an important role in detection and detection, the dead time of the dead is generally the time of the criminal elements, determining the time can play a role in excluding, confirming the suspect and so on. After death, because of the continuous activation of DNA inscribe, the number of DNA will be continuously decreased with the extension of time, and will present a certain regularity. By mastering the law, the technician can determine the exact time of death by using modern science and technology to test the number of DNA in the dead. In addition, with the development of biological science and technology, the application of forensic DNA testing technology in the investigation is no longer limited to the inspection of biological samples such as human tissues and body fluids. This technique has been used in the identification of other biological evidence, through the analysis of the insect DNA on the body can identify the species of insects, and then through the analysis of the morphological characteristics of the various growth stages, according to the morphological changes of the growth and development can determine the dead time of the dead, so as to provide evidence and clues for the investigation.

Provide the basis for Identifying the Offender by Examining the DNA of the Biological Samples Left Behind by the Suspect. The criminal suspect is identified by the same identification of the body fluid, hair, semen and other biological samples obtained from the crime scene and the biological samples obtained from the suspect.

\section{The Problems in the Application of Forensic DNA Evidence}

Some Investigators Work Attitude is not Serious, the Collection Ability is Low. The forensic DNA test should first be collected from the site to biological samples, such as blood, hair, semen, saliva, vaginal secretions, urine, skin tissue, and so on. The material evidence is often left in the difficult to detect, can collect these biological samples, the work attitude of the site investigators is very important, some staff are not serious in the site investigation, which leads to the lack of samples. Some staff members lack the basic knowledge of forensic DNA forensic examination, and are unaware of the physical evidence available on the site for DNA testing, such as chewing gum, sputum, cigarette butts, etc., thus missing the collection of samples. In addition, in some cases, after the crime investigators failed to effectively protect the crime scene, resulting in the crime scene material evidence was destroyed, affecting the collection of samples.

The Sample Material is Polluted or Influenced by the External Environment, and the Success Rate of the Detection Result is Reduced. The PCR - str technique is characterized by very high sensitivity and slight contamination of the samples. the DNA fragments of the pollutants are multiplied by the geometric series, resulting in the mixing of DNA in the samples, which directly affects the interpretation of the identification results. The factors causing pollution to the samples are: improper packing of objects containing trace biological samples; The material is mixed with other biological material or other non - biological material; The field investigators collect the samples without gloves, and have a cough for the samples. In addition to contamination, forensic DNA samples can be degraded by the effects of the surrounding environment. For example, if the material is not sent to the test in time, it will be affected by sunlight ( the integrity of the DNA molecule is affected by the ultraviolet radiation ), the bacteria ( the bacterium inscribe can directly damage the human DNA molecules, and the degradation of DNA ), and so on, so that the forensic DNA test results are affected, and its proof strength is weakened.

There are Problems in the Evidence Collection Procedures of the Samples. DNA testing has a certain dependence. apart from the trace samples collected from the field, the role of DNA in the investigation needs to be carried out in a range of personnel, such as blood extraction, urine 
extraction, hair extraction and so on, so as to identify or exclude the criminals in comparison with the DNA test results of physical evidence in the field. However, in the investigation work, because of the psychological exclusion of the sampling behavior and the criminal suspect guilty of a guilty conscience for evading detection purposes, the people who are asked to sample often do not cooperate with the attitude. In these cases, investigators often rely on the help of local governments to borrow some legitimate rather than the reasons for the investigation needs, or sometimes take a direct coercive sampling of the suspect. As there is no relevant provision in the criminal procedure law of our country, it only stipulates that the investigator can make compulsory inspection on the suspect when he refuses the physical examination, and the investigator has no legal basis or even violates the basic human rights of the citizen to some extent by referring to the practice of compulsory or not informing the real purpose. The illegality of this procedure directly affects the use of DNA evidence in court.

There are Problems in the DNA Identification Process of Biological Samples. The application of forensic DNA testing technology in criminal proceedings has solved many problems that can not be solved before, and played an incomparable role in the investigation and detection of cases. DNA test results as a judicial authentication conclusion, its inspection process has a certain degree of professionalism, it will involve laboratory inspectors, inspection methods, inspection procedures, inspection equipment, and so on, all of these factors may affect the accuracy of DNA test results.

The Results of DNA Testing have Problems in the Application of Criminal Trials. The production and application of forensic DNA evidence is operated by people, so its scientific and accuracy is not 100 percent. However, in the judicial practice, many judicial personnel are superstitious to the accuracy of DNA evidence, generally will determine its evidential value, ignore the defense of the defense, sometimes lead to the occurrence of enemy cases.

\section{Suggestions on Improving the Application of DNA Evidence in the Administration of Justice}

Strengthening Procedural Safeguards for Forensic DNA Evidence. First, the extraction subject of DNA evidence samples is required by specific personnel, and experts believe that it should be required to have a considerable degree of education and extensive validation experience familiar with and understand the principles of DNA testing, clarify the scope and limitations of DNA analysis methods, and understand the procedures related to DNA testing. These personnel can extract the samples according to the needs of the identification and carry out proper classification and packaging to prepare for future identification. Secondly, to provide for the steps to be prepared before the extraction of evidence. In order to ensure the smooth progress of the collection of biological samples, before the formal extraction, the relevant preparations need to be done to ensure the efficiency of the extraction of biological samples and to avoid human contamination of the samples. These preparatory steps should include: discussion and assessment of the crime scene situation; Arranging the person to collect the material on site; Prepare the form required for site survey; Prepare to extract the cleansing tools and other materials required for the samples. Again, the witness and records of the field samples are required. In order to ensure forensic DNA evidence has the ability to evidence, in the extraction of biological samples, need to be witnessed. When each piece of evidence is extracted at the site, it is to be sealed in the presence of the witness and signed by the witness. Finally, because the samples are susceptible to degradation and contamination by the environmental impact, when the biological samples containing DNA are extracted, they are sent to the identification mechanism at the fastest speed, so that the on-site samples will begin to transfer across time and across space. The process of transfer, custody, opening and unpacking shall be completed jointly by the investigators and custodians, and the integrity and authenticity of the seal shall be checked at each step, and a detailed record shall be made to be signed by the actor, so as to ensure the authenticity and reliability of the seal.

Improve the Legal Provisions of Sample Sampling of Forensic DNA Testing. The forensic DNA evidence is the same conclusion obtained by the forensic examiners after the examination of the biological samples of the crime scene and the biological samples of the suspect. This means that 
in the process of DNA testing, it is necessary to involve human samples such as blood, saliva, semen, urine, feces, hair, pubic hair and other samples containing human nuclear cell substances. Because the DNA test results contain a lot of personal information, such as improper handling of the leakage of these information will violate the privacy of citizens, so in the actual work sample collection will be rejected. With the improvement of the role of DNA evidence in criminal cases, it is necessary to collect DNA samples. In order to protect citizens' rights and provide legal basis for the compulsory sampling of investigators, we should make strict regulations on the subject, object, measurement, scope of collection, the preservation of samples, the destruction of samples, the confidentiality of information, etc.

Improving the Procedure for the Examination of DNA Evidence in the Tribunal. The results of DNA test as a conclusion of judicial authentication is an indirect evidence. Like other types of circumstantial evidence, it can only prove one aspect of the case, and must form a complete chain of evidence with other evidence in order to meet the criteria of the case. Therefore, the judicial personnel should have the correct understanding of DNA evidence, can not blindly believe in its accuracy. Each forensic DNA evidence must be cross-examined by the prosecution and the defence in the court. This requires the court in the trial process must establish a case handling police to testify the system, improve the appraiser to testify in court system, let the police and the appraiser to accept the prosecution and the defense of the two sides to ensure the impartiality and scientific conclusion of the conclusion.

Establishment of a DNA Database. DNA database plays an important role in criminal proceedings. After the crime of Cocu is established, when the case occurs, the test results of the field biological samples are compared with the information stored in the criminal records library, and the criminals can be identified when they reach the unified identification. Through the establishment of the site library, the field samples DNA typing is compared with the data in the field database, and the two can carry on the string and investigate the case. In addition, by establishing the DNA database of the parents of missing persons, the DNA test data of the unknown body can be compared with the DNA database of the parents of missing persons in order to confirm the identity of the deceased when the unknown body and the body of the body are identified. In practice, if the national DNA database can be established and the sharing of resources can be realized, the forensic DNA technology will be more proactive in providing evidence for the detection and identification of the perpetrators.

\section{Conclusion}

Since the introduction of DNA technology into the field of forensic science, it has gradually become the main means of forensic evidence identification in criminal cases. This paper focuses on the principle of DNA technology and the specific application in criminal cases. At the same time, the existing problems in the application of DNA evidence are pointed out. Finally, the rationalization proposals are given, which greatly promote the scientific application of DNA evidence.

\section{References}

[1]TsukadaK,

TakayanagiK,

AsamuraH, etal. Multiplexshorttandemrepeattypingindegraded samplesusingnewlydesignedprimersfortheTH01, TPOX, CSF1PO, andvWAloci[J].LegMed（Tokyo）, 2002, 4 (4) 239-245.

[2]GillP,

WhitakerJ

FlaxmanC, etal.AninvestigationoftherigorofinterpretationrulesforSTRsderivedfromlessthan100pgofDNA[J].ForensicSciI nt, 2000, 112 (1) : 17-40

[3]HansonEK, BallantyneJ.Wholegenomeamplificationstrategyforforensicgeneticanalysisusingsingleorfewcell equivalentsof genomicDNA[J].AnalBiochem, 2005, 346 (2) : 246-257.

[4]B.Wang.The technology application of DNA typing technology in forensic science, the biotechnology world,2013. 
[5]H.W.Dang,J.Mao,H.Wang.Current status and progress of forensic DNA testing in difficult biological samples [ $\mathrm{J}$ ]. journal of forensic medicine, 2012, 28 (1) : 52-54.

[6] Z.B.Xu, The application and prospect of DNA analysis technology in forensic science, journal of law and medicine, 2000. 